\title{
Helmholtz-Manakov solitons
}

\section{J. M. Christian, G. S. McDonald and P. Chamorro-Posada ${ }^{\dagger}$}

Joule Physics Laboratory, School of Computing, Science and Engineering, Institute for Materials Research, University of Salford, Salford M5 4WT, U.K.

$\dagger$ Departmento de Teoría de la Señal y Comunicaciones e Ingeniería Telemática, Universidad de Valladolid, ETSI Telecomunicación, Campus Miguel Delibes s/n, 47011 Valladolid, Spain.

Accepted in Physical Review E, $21^{\text {st }}$ September 2006

\begin{abstract}
A novel spatial soliton-bearing wave equation is introduced, the Helmholtz-Manakov (H-M) equation, for describing the evolution of broad multi-component self-trapped beams in Kerr-type media. By omitting the slowly-varying envelope approximation, the $\mathrm{H}-\mathrm{M}$ equation can describe accurately vector solitons propagating and interacting at arbitrarily large angles with respect to the reference direction. The H-M equation is solved using Hirota's method, yielding four new classes of Helmholtz soliton that are vector generalizations of their scalar counterparts. General and particular forms of the three invariants of the H-M system are also reported.
\end{abstract}

PACS numbers: 42.65.Tg (optical solitons), 42.79.Ta (optical computers), 94.05.Fg (Solitons and solitary waves), 05.45.Yv (solitons, nonlinear dynamics of) 


\section{INTRODUCTION}

Vector solitons are well known in Optics $[1,2]$. They are multi-component, localized structures that can become dominant modes of a system when linear broadening effects are offset by non-linearity. During pulse propagation, on the one hand, vector solitons can arise when dispersion is balanced by self- and cross-phase modulation. Spatial soliton beams, on the other, can result if diffractive spreading is opposed by medium self- and mutual-focusing. Many years ago, Manakov [3] proposed a vector extension of the scalar Non-Linear Schrödinger (NLS) equation [4] to describe multi-component pulse/beam evolution in the presence of positive Kerr non-linearity. Using inverse-scattering theory, he was able to derive an exact analytical 2component sech-type soliton solution.

In birefringent optical fibres, vector solitons play a central role in describing polarization-division multiplexing (PDM) configurations, where light coupled into the fibre is polarized along two transverse orthogonal axes [5]. Over sufficiently long distances, birefringence can average out stochastically to zero, and the model capturing evolution is the (temporal) Manakov equation [6]. In spatial contexts, multicomponent solutions for a defocusing (negative) Kerr non-linearity have been reported [7]. These new solutions have a more complicated (topological) structure than their more familiar sech-type counterparts in the focusing regime [3]. Manakov-like solitons, comprising two orthogonal transverse field components, have been observed in birefringent Kerr-type planar waveguides [8]. Multi-component spatial solitons in $\chi^{(2)}$ [9] and $\chi^{(3)}$-like [10] photorefractive media have also received attention, and new types of structures such as multi-hump [11] and holographic [12] solitons have been predicted. 
Recently, it has been shown that collisions between Manakov solitons are inelastic, and that there is a redistribution of energy between the interacting components [13]. Such an intrinsic effect has no analogue in scalar (i.e. NLS) theory, where the energy and momentum of each constituent soliton is preserved [4]. It has been suggested [14] that this unique property of the Manakov system could provide the basis for optical computing with solitons [15]. The energy-exchange process was found to depend critically upon the angle between the interacting beams. It is therefore desirable to have a model capable of capturing arbitrary angles.

The main interest in this Article lies with the oblique propagation of multicomponent spatial solitons in Kerr planar waveguides. In this geometry, there is a longitudinal (reference) and a single effective transverse dimension that, in uniform media, are physically equivalent. We propose the novel Helmholtz-Manakov equation as a 2-component generalization of the scalar Non-Linear Helmholtz (NLH) equation [16]. This model is appropriate for capturing the propagation [17] and interaction [18] of broad vector-soliton beams at arbitrary angles with respect to the reference direction. Here, we present four new families of exact analytical soliton solution to the H-M equation, and derive the corresponding conservation laws.

\section{HELMHOLTZ NON-PARAXIALITY}

The NLH and H-M equations provide a full description of oblique (off-axis) evolution for broad optical beams of moderate intensity [16-18]. Under these conditions, the polarization-scrambling term in Maxwell's equations can be neglected [1921] and this leads to two important simplifications: (i) in uniform media, the associated refractive-index distributions may be treated within the scalar approximation, and 
(ii) the electric field may be regarded as being purely transverse (we can ignore the longitudinal component). For NLH solitons, one typically considers a TE mode polarized in the plane of the waveguide [18]. For vector solitons, the electric field can have two orthogonal polarization components [3]. In this particular context, the H-M equation is a valid model when birefrigence can be neglected [22]. Alternatively, the H-M model can describe the interplay between two incoherently-coupled fields sharing the same polarization state where, typically, both are TE modes [18]. This latter consideration could be extended to the case of $N$ components [23].

The oblique evolution of broad beams context defines the Helmholtz nonparaxial scenario [16-18], which is physically and mathematically distinct from the narrow-beam regime considered by other authors. Perturbative narrow-beam corrections to the governing equation [19-21,24], derived from single-parameter order-ofmagnitude analyses of Maxwell's equations, are both unnecessary (we consider only broad beams) and invalid (off-axis effects are not quantified solely by a single small parameter) in Helmholtz non-paraxality. The accurate description of obliquelypropagating and interacting beams, relative to the reference direction, requires one to respect the equivalence of the transverse and longitudinal dimensions in uniform media. This is achieved by avoiding the slowly-varying envelope approximation (SVEA); NLH and H-M models then arise as natural governing equations [17,18].

In a normalized form, the $\mathrm{H}-\mathrm{M}$ equation is given by

$$
\kappa \frac{\partial^{2} \mathbf{U}}{\partial \zeta^{2}}+i \frac{\partial \mathbf{U}}{\partial \zeta}+\frac{1}{2} \frac{\partial^{2} \mathbf{U}}{\partial \xi^{2}} \pm\left(\mathbf{U}^{\dagger} \mathbf{U}\right) \mathbf{U}=\mathbf{0}
$$


where the \pm sign flags a focusing $(+)$ or a defocusing $(-)$ Kerr non-linearity. $\xi=\sqrt{2} x / w_{0}$ and $\zeta=z / L_{D}$ are the scaled transverse and longitudinal coordinates, normalized to the waist $w_{0}$ and diffraction length $L_{D}=k w_{0}^{2} / 2$ of a reference Gaussian beam, respectively. The column vector $\mathbf{U}$ is the dimensionless electric field in $\mathbf{E}(x, z)=E_{0} \mathbf{U}(x, z) \exp (i k z)$, where $k=2 \pi n_{0} / \lambda, \lambda$ is the optical wavelength, $n_{0}$ is the linear refractive index at the optical frequency, $E_{0}=\left(n_{0} / k\left|n_{2}\right| L_{D}\right)^{1 / 2}$ and $n_{2}$ is the Kerr coefficient. $\quad \kappa=1 /\left(k w_{0}\right)^{2}=\left(\lambda / w_{0}\right)^{2} / 4 \pi^{2} n_{0}^{2}$ is the inverse beam-width parameter. In the case of two field components, one has that $\mathbf{U}=[A, B]^{T}$, where $T$ denotes the transpose, and $\mathbf{U}^{\dagger}$ is the Hermitian adjoint of $\mathbf{U}$. A further generalization to $N$ components then involves including further entries in $\mathbf{U}$.

The restoration of spatial symmetry in the governing equation [17] leads to several novel features absent from paraxial theory. Firstly, there is no physical distinction between transverse $(x)$ and longitudinal $(z)$ coordinates and light is allowed to diffract in both these dimensions. Secondly, inclusion of $\kappa \partial_{\zeta \zeta}$ leads to a dispersion relation that supports both travelling- and standing-wave solutions [18]. This is in contrast to the NLS [4] and Manakov [3] equations, where the SVEA breaks the symmetry between not only $x$ and $z$, but also between $+z$ and $-z$, (backward-travelling waves are not supported). Thus, paraxial wave equations are subjected to the physical limitation of describing beams that are either axial or very nearly axial.

The inherent bi-directionality of NLH-based models leads to angular beam broadening (see Fig.1). This effect arises from considering two descriptions of the 
same beam from different frames of reference, and is a requirement based on geometry [16]. The Helmholtz non-paraxial formalism also allows for a well-defined connection between the soliton velocity $V$ in the $(\xi, \zeta)$ frame and the propagation angle $\theta$ (relative to the $z$ axis) in the unscaled $(x, z)$ frame through [16-18]

$$
\tan \theta=\sqrt{2 \kappa} V
$$

Equation (2) verifies that the (purely geometrical) Helmholtz correction $2 \kappa V^{2}$ may assume an arbitrarily large value, even for beams with $\kappa \simeq 0$. During off-axis evolution, one can have a regime where $2 \kappa V^{2} \sim O(1)$, while simultaneously respecting $\kappa \ll O(1)$ because the beam is always broad. This possibility demonstrates that descriptions based solely upon $\kappa$-type expansions of Maxwell's equations are inappropriate for capturing the angular type of non-paraxiality [19-21,24]. Indeed, the dominant Helmholtz correction to paraxial theory, embodied in Eq. (2), is determined solely by the beam's propagation angle and can be of any order irrespective of the size of $\kappa$.

\section{HELMHOLTZ-MANAKOV SOLITONS}

\section{A. Solitons in self-focusing Kerr media}

For a focusing Kerr medium, the simplest case to consider is that of a vector beam with two forward-propagating constituent components, each with a symmetric sech-type profile. The most straightforward method of obtaining such solutions is to use an ansatz approach to seek the on-axis solution, and then apply a rotational transformation [25] to seek the more general off-axis beam. The resulting bright-bright HM solution is 


$$
\begin{aligned}
& \mathbf{U}(\xi, \zeta)=\mathbf{C} \eta \operatorname{sech}\left[\frac{\eta(\xi+V \zeta)}{\sqrt{1+2 \kappa V^{2}}}\right] \exp \left[i \sqrt{\frac{1+2 \kappa \eta^{2}}{1+2 \kappa V^{2}}}\left(-V \xi+\frac{\zeta}{2 \kappa}\right)\right] \exp \left(-i \frac{\zeta}{2 \kappa}\right) \\
& \text { where, } \quad \mathbf{C}=\left[\begin{array}{c}
e^{i \delta_{1}} \cos (\alpha) \\
e^{i \delta_{2}} \sin (\alpha)
\end{array}\right]
\end{aligned}
$$

$\mathbf{C}$ is a complex column vector obeying $\mathbf{C}^{\dagger} \mathbf{C}=1$, while $\eta$ defines the amplitude and $V$ the transverse velocity. The free parameter $\alpha$ determines the strength of the excitation in each component, and $\delta_{j}(j=1,2)$ are the component phases. The choice of values of $\alpha$ and the $\delta_{j}$ 's allows a wide variety of soliton states to be constructed.

The second class of vector beam that a focusing Kerr medium can support is the bright-dark soliton, for which the forward-propagating solution is

$$
\begin{array}{r}
A(\xi, \zeta)=\sqrt{B_{0}^{2} \cos ^{2} \phi+a^{2}} \operatorname{sech}\left[\frac{a(\xi+W \zeta)}{\sqrt{1+2 \kappa W^{2}}}\right] \\
\times \exp \left[i \sqrt{\frac{1+2 \kappa\left(a^{2}+2 \chi^{2}\right)}{1+2 \kappa W^{2}}}\left(-W \xi+\frac{\zeta}{2 \kappa}\right)\right] \exp \left(-i \frac{\zeta}{2 \kappa}\right), \\
B(\xi, \zeta)=B_{0}\left[\cos \phi \tanh \left(\frac{a(\xi+W \zeta)}{\sqrt{1+2 \kappa W^{2}}}\right)+i \sin \phi\right] \\
\quad \times \exp \left[i \sqrt{\frac{1+4 \kappa \chi^{2}}{1+2 \kappa V^{2}}}\left(-V \xi+\frac{\zeta}{2 \kappa}\right)\right] \exp \left(-i \frac{\zeta}{2 \kappa}\right),
\end{array}
$$

This structure comprises a Helmholtz bright soliton [25] in one component, and a dark-type topological excitation in the other. The tanh component (4b) is generally grey, and has an intrinsic velocity,

$$
V_{0}=\frac{a \tan \phi}{\sqrt{1+2 \kappa\left(2 \chi^{2}-a^{2} \tan ^{2} \phi\right)}} .
$$


This velocity depends upon both the non-paraxial parameter $\kappa$ and the plane-wave background intensity $\chi^{2} \equiv B_{0}^{2}$. When $B_{0} \rightarrow 0$, the dark component is null and one recovers the Helmholtz Kerr scalar bright soliton [25]. The net velocity $W$ of the vector beam (4) is then given by

$$
W=\frac{V-V_{0}}{1+2 \kappa V V_{0}} .
$$

As in the case of the scalar Helmholtz dark soliton [26], $W$ has the physical interpretation of velocity summation in the unscaled reference frame.

\section{B. Solitons in defocusing Kerr media}

When seeking a general dark soliton of the defocusing NLH equation [26], an ansatz approach cannot determine the full solution (geometrical considerations also have be to made). Similar difficulties arise in the vector case. Thus, Hirota's method [27] has been used to solve the defocusing H-M equation. The forward-propagating dark-bright vector soliton solution is given by

$$
\begin{array}{r}
A(\xi, \zeta)=A_{0}\left[\cos \phi \tanh \left(\frac{a(\xi+W \zeta)}{\sqrt{1+2 \kappa W^{2}}}\right)+i \sin \phi\right] \\
\quad \times \exp \left[i \sqrt{\frac{1-4 \kappa \chi^{2}}{1+2 \kappa W^{2}}}\left(-V \xi+\frac{\zeta}{2 \kappa}\right)\right] \exp \left(-i \frac{\zeta}{2 \kappa}\right), \\
B(\xi, \zeta)=\sqrt{A_{0}^{2} \cos ^{2} \phi-a^{2}} \operatorname{sech}\left(\frac{a(\xi+W \zeta)}{\sqrt{1+2 \kappa W^{2}}}\right) \\
\times \exp \left[i \sqrt{\frac{1+2 \kappa\left(a^{2}-2 \chi^{2}\right)}{1+2 \kappa W^{2}}}\left(-W \xi+\frac{\zeta}{2 \kappa}\right)\right] \exp \left(-i \frac{\zeta}{2 \kappa}\right) .
\end{array}
$$

The grey component (7a) has an intrinsic velocity

$$
V_{0}=\frac{a \tan \phi}{\left[1-2 \kappa\left(2 \chi^{2}+a^{2} \tan ^{2} \phi\right)\right]^{1 / 2}},
$$


where $\chi \equiv A_{0}^{2}$, and the net velocity $W$ is given by Eq. (6). The dark-bright soliton is constrained by the condition $A_{0}^{2} \cos ^{2} \phi \geq a^{2}$. When the equality is satisfied, the bright component vanishes and the remaining component (i.e., the primary component) recovers an exact non-paraxial dark soliton [26].

The bright-dark and dark-bright solutions are characterized by three velocities, $V, V_{0}$ and $W$, which are associated with the propagation angles $\tan \theta=\sqrt{2 \kappa} V$, $\tan \theta_{0}=\sqrt{2 \kappa} V_{0}$ and $\tan \left(\theta-\theta_{0}\right)=\sqrt{2 \kappa} W$ [20] (note that any two velocities can be chosen independently). However, it is important to note that these solutions are not equivalent. When the dark components have the same parameters (i.e. background intensities and values of $\kappa, a$ and $\phi$ ), the soliton intensity profiles different slightly from one another due to their different intrinsic velocities, given by Eqs. (5) and (8), respectively (see Fig. 1). Another distinction between dark-bright and bright-dark solutions is that the primary component (that which can propagate stably when the other component is not excited) is a dark soliton in the former, and a bright soliton in the latter.

The secondary component of these mixed-pair (that is, bright-dark and darkbright) beams possesses anti-guiding properties, a phenomenon seen also with Manakov solitons [7]. For the dark-bright soliton, the bright (secondary) component, propagating in the waveguide induced by the dark (primary) component, has an intensity maximum at its centre. The presence of such a maximum in a defocusing medium leads to a lowering of the refractive index at the beam centre, thus weakening the overall guiding effect. The subsequent propagation can be thought of as a com- 
petitive process between the non-linear refractive index changes brought about by the individual dark and bright components. When these processes are balanced precisely, an equilibrium state is formed and a vector soliton may propagate. These refractive index changes are reversed for the bright-dark soliton in a focusing medium, but the underlying physics remains the same.

The (forward-propagating) dark-dark vector soliton of the defocusing $\mathrm{H}-\mathrm{M}$ equation has also been found using Hirota's method

$$
\begin{array}{r}
A(\xi, \zeta)=A_{0}\left[\cos \phi_{1} \tanh \left(\frac{a(\xi+W \zeta)}{\sqrt{1+2 \kappa W^{2}}}\right)+i \sin \phi_{1}\right] \\
\times \exp \left[i \sqrt{\frac{1-4 \kappa \chi^{2}}{1+2 \kappa V_{1}^{2}}}\left(-V_{1} \xi+\frac{\zeta}{2 \kappa}\right)\right] \exp \left(-i \frac{\zeta}{2 \kappa}\right), \\
\begin{aligned}
& B(\xi, \zeta)=B_{0}\left[\cos \phi_{2} \tanh \left(\frac{a(\xi+W \zeta)}{\sqrt{1+2 \kappa W^{2}}}\right)+i \sin \phi_{2}\right] \\
& \quad \quad \exp \left[i \sqrt{\frac{1-4 \kappa \chi^{2}}{1+2 \kappa V_{2}^{2}}}\left(-V_{2} \xi+\frac{\zeta}{2 \kappa}\right)\right] \exp \left(-i \frac{\zeta}{2 \kappa}\right),
\end{aligned}
\end{array}
$$

where $\chi^{2} \equiv A_{0}^{2}+B_{0}^{2}$ is the total (incoherent) intensity of the vector beam. The expressions for the intrinsic velocities are

$$
V_{0 j}=\frac{a \tan \phi_{j}}{\left[1-2 \kappa\left(2 \chi^{2}+a^{2} \tan ^{2} \phi_{j}\right)\right]^{1 / 2}},
$$

where $j=1,2$, and there is a dependence not only on the non-paraxial parameter $\kappa$ but also on $\chi^{2}$. The soliton parameters are connected by the implicit relationship $A_{0}^{2} \cos ^{2} \phi_{1}+B_{0}^{2} \cos ^{2} \phi_{2}=a^{2}$ that removes a degree of freedom from the system. The two components necessarily have the same net velocity $W$, but their plane-wave back- 
grounds may travel in different directions. Note that Eq. (9) for the dark-dark soliton is formally identical to Eq. (8) for the dark-bright solution.

Paraxial solitons of the NLS [4] and Manakov [3] equations can have arbitrarily large values of phase angle $\phi<\pi / 2$. In contrast, H-M solitons possess a maximum "greyness" denoted by $\phi_{\max }$. For the defocusing non-linearity, $\phi_{\max }$ is defined by

$$
\tan \phi_{\max }=\sqrt{\frac{1-4 \kappa \chi^{2}}{2 \kappa a^{2}}}
$$

where $\chi^{2} \equiv A_{0}^{2}$ for the dark-bright soliton, and $\chi^{2} \equiv A_{0}^{2}+B_{0}^{2}$ in the dark-dark case. A similar expression can be derived for the bright-dark solution (4). This limit corresponds to a physical constraint on the largest intrinsic velocity that a dark soliton may possess, depending on the background intensity. When $\phi \rightarrow \phi_{\max }$, the intrinsic velocity diverges and the dark component propagates in a direction perpendicular to that of the background plane wave, $\theta_{0} \rightarrow \pm \pi / 2$ [26].

\section{CONSERVED QUANTITIES}

Knowledge of the invariants is of fundamental importance. They are also useful tools for testing the integrity of any numerical scheme used in computer simulations [28]. The H-M equation (1) may be regarded as the Euler-Lagrange equation of motion corresponding to a Lagrangian density $L$, from which one defines a pair of canonically-conjugate momentum variables, $\pi$ and $\tilde{\pi}$ :

$$
L\left(\mathbf{U}, \mathbf{U}^{\dagger}\right)=\frac{i}{2}\left(\mathbf{U}^{\dagger} \frac{\partial \mathbf{U}}{\partial \zeta}-\frac{\partial \mathbf{U}^{\dagger}}{\partial \zeta} \mathbf{U}\right)-\kappa \frac{\partial \mathbf{U}^{\dagger}}{\partial \zeta} \frac{\partial \mathbf{U}}{\partial \zeta}-\frac{1}{2} \frac{\partial \mathbf{U}^{\dagger}}{\partial \xi} \frac{\partial \mathbf{U}}{\partial \xi}+\frac{1}{2}\left(\mathbf{U}^{\dagger} \mathbf{U}\right)^{2},
$$

whereby 


$$
\pi \equiv \frac{\partial L}{\partial \mathbf{U}_{\zeta}}=-\left(\frac{i}{2}+\kappa \frac{\partial}{\partial \zeta}\right) \mathbf{U}^{\dagger}, \quad \tilde{\pi} \equiv \frac{\partial L}{\partial \mathbf{U}_{\zeta}^{\dagger}}=\left(\frac{i}{2}-\kappa \frac{\partial}{\partial \zeta}\right) \mathbf{U},
$$

and $\mathbf{U}_{\zeta} \equiv \partial_{\zeta} \mathbf{U}$. It is then straightforward to calculate the conserved quantities [29]. The fundamental quantity is the energy-flow $E$, which arises from invariance of $L$ under a global phase transformation. The second conserved quantity is the linear momentum $M$ of the system, found from invariance under an infinitesimal translation in $\xi$. The third conserved quantity is the Hamiltonian $H$, derived from consideration of translations in the evolution variable, $\zeta$.

$$
\begin{gathered}
E=\int_{-\infty}^{+\infty} d \xi\left[\mathbf{U}^{\dagger} \mathbf{U}-i \kappa\left(\mathbf{U}^{\dagger} \frac{\partial \mathbf{U}}{\partial \zeta}-\frac{\partial \mathbf{U}^{\dagger}}{\partial \zeta} \mathbf{U}\right)\right] \\
M=\int_{-\infty}^{+\infty} d \xi\left[\frac{i}{2}\left(\mathbf{U}^{\dagger} \frac{\partial \mathbf{U}}{\partial \xi}-\frac{\partial \mathbf{U}^{\dagger}}{\partial \xi} \mathbf{U}\right)-\kappa\left(\frac{\partial \mathbf{U}^{\dagger}}{\partial \zeta} \frac{\partial \mathbf{U}}{\partial \xi}+\frac{\partial \mathbf{U}^{\dagger}}{\partial \xi} \frac{\partial \mathbf{U}}{\partial \zeta}\right)\right], \\
H=\int_{-\infty}^{+\infty} d \xi\left[\frac{1}{2} \frac{\partial \mathbf{U}^{\dagger}}{\partial \xi} \frac{\partial \mathbf{U}}{\partial \xi}-\kappa \frac{\partial \mathbf{U}^{\dagger}}{\partial \zeta} \frac{\partial \mathbf{U}}{\partial \zeta}-\frac{1}{2}\left(\mathbf{U}^{\dagger} \mathbf{U}\right)^{2}\right] .
\end{gathered}
$$

Equations (13)-(15) also apply to the scalar NLH equation, by allowing one of the field components to be null. For bright-bright H-M solitons, it is found that:

$$
\begin{gathered}
E=2 \eta \sqrt{1+2 \kappa \eta^{2}} \\
M=\frac{2}{3} \eta V \frac{3+4 \kappa \eta^{2}}{\sqrt{1+2 \kappa V^{2}}}, \\
H=\frac{\eta}{\kappa} \sqrt{1+2 \kappa \eta^{2}}-\frac{\eta}{3 \kappa} \frac{3+4 \kappa \eta^{2}}{\sqrt{1+2 \kappa V^{2}}} .
\end{gathered}
$$

These results are exactly the same as for the scalar bright Helmholtz soliton [25]. As expected they reduce to their paraxial counterparts in the multiple limit $\kappa \rightarrow 0$, $\kappa \eta^{2} \rightarrow 0$ and $\kappa V^{2} \rightarrow 0[1,2]$. It is interesting to note that $\partial H / \partial M=V$ (as in the paraxial case). For other classes of H-M soliton, possessing at least one dark compo- 
nent, the invariant integrals (13)-(15) can be strongly divergent. A renormalization procedure is thus necessary to remove such infinities.

\section{CONCLUSION}

In this Article, we have considered broad multi-component spatial beams in uniform planar waveguides, placing particular emphasis on the inherent spatial symmetry of such systems. The novel Helmholtz-Manakov equation, which generalizes our earlier work on scalar beams $[16-18,25,26,30]$, has been introduced and its 2component solutions (localized, mutually-trapped structures) have been derived using Hirota's method. The new solutions uncover explicit physical dependencies of novel quantitative and qualitative features. In a following publication, we will present the results of a perturbative analysis, which has tested and verified the stability properties of each new soliton family.

We expect the H-M equation and its soliton solutions to be relevant in other optical contexts, such as photorefractives [9,10], multi-hump [11] and holographic [12] solitons. It is also likely to provide a key analytical platform in the understanding of vector-soliton interactions (both co- and counter-propagating scenarios) at arbitrarily large angles [18]. We expect new doors of investigation to be opened by lifting the angular restrictions of current paraxial models. This has particular importance, for example, in the field of optical computing [14,15]; it may also find application in optical contexts involving feedback [31]. Helmholtz generalizations offer broad physical insight into a wide variety of angular geometries by taking full account of the role spatial symmetry plays in diffraction/self-focusing processes. 


\section{ACKNOWLEDGEMENTS}

The authors would like to acknowledge financial support from EPSRC sponsorship and the University of Salford. 


\section{FIGURE CAPTIONS}

FIG. 1 (color on-line). Characteristic angular beam-broadening effects associated with Helmholtz solitons for (a) bright-dark, and (b) dark-bright cases. Solid line: $|A(\xi, 0)|$; dashed line: $|B(\xi, 0)|$. Dotted lines correspond to paraxial solutions. For a non-paraxial parameter $\kappa=10^{-4}$, a transverse velocity of $V \approx 70.71$ yields a physical propagation angle $\theta=45^{\circ}$ so that $2 \kappa V^{2}=O(1)$ and one has a strongly non-paraxial regime. Other solution parameters are $a=0.5, \phi=\pi / 6, B_{0}=1$ [in (a)] and $A_{0}=1$ [in (b)]. 
FIGURE 1

J. M. Christian et al.

Submitted to Physical Review E
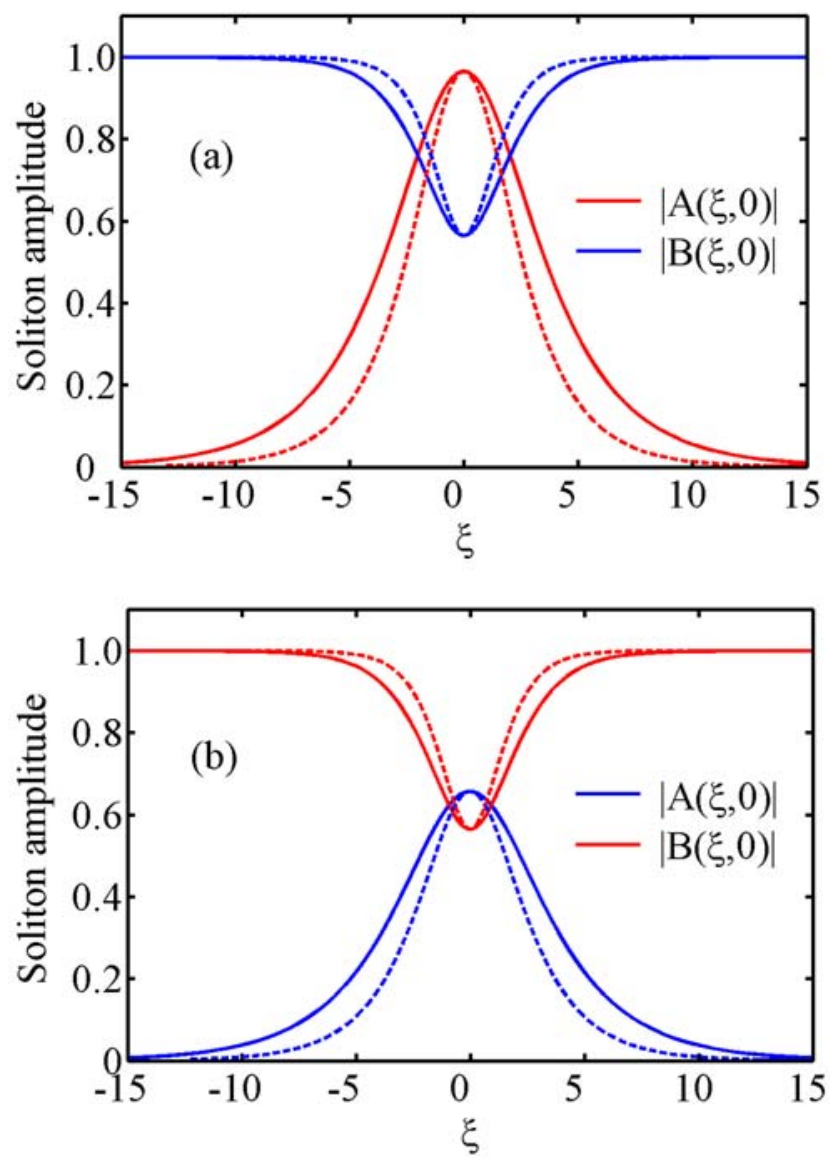
[1] Y. S. Kivshar, Opt. Quant. Elect. 30, 517 (1998).

[2] Y. S. Kivshar and B. Luther-Davies, Phys. Rep. 298, 81 (1998).

[3] S. V. Manakov, Sov. Phys. JETP 38, 248, (1974).

[4] V. E. Zakharov and A. B. Shabat, Sov. Phys. JETP 34, 62 (1972); 37, 823 (1973).

[5] C. R. Menyuk, J. Opt. Soc. Am. B 5, 392 (1988); IEEE J. Quantum Electron. QE-23, 174 (1987); Opt. Lett. 12, 614 (1987).

[6] J. Yang, Phys. Rev. E 65, 036606 (2002); 64, 026607 (2001).

[7] A. P. Sheppard and Y. S. Kivshar, Phys. Rev. E 55, 4773 (1997).

[8] J. U. Kang, G. I. Stegeman, J. S. Aitchison, and N. N. Akhmediev, Phys. Rev. Lett. 76, 3699 (1996); J. U. Kang, G. I. Stegeman and J. S. Aitchison, Opt. Lett. 21, 189 (1996).

[9] C. Hou and L. Wang, Optik 115, 405 (2004); D. N. Christodoulides, S. R. Singh, M. I. Carvalho, and M. Segev, Appl. Phys. Lett. 681763 (1996); Z. Chen, M. Segev, T. Coskun, and D. N. Christodoulides, Opt. Lett. 21, 1426 (1996).

[10] C. Hou, Z. Zhou and X. Sun, Optical Materials 27, 63 (2004).

[11] E. A. Ostrovskaya and Y. S. Kivshar, J. Opt. B: Quant. Semiclass. Opt. 1, 77 (1999); E. A. Ostrovskaya, Y. S. Kivshar, D. V. Skryabin, and W. J. Firth, Phys. Rev. Lett. 83, 296 (1999).

[12] J. R. Salgueiro, A. A. Sukhorukov, and Y. S. Kivshar, Opt. Lett. 28, 1457 (2003); O. Cohen et al., Opt. Lett. 27, 2031 (2002).

[13] R. Radhakrishnan, M. Lakshmanan and J. Hietarinta, Phys. Rev. E 56, 2213 (1997).

[14] C. Anastassiou et al., Phys. Rev. Lett. 83, 2332 (1999). 
[15] K. Steiglitz, Phys. Rev. E 63, 016608 (2003); M. H. Jakubowski, K. Steiglitz, and R. Squier, Phys. Rev. E 58, 6752 (1998).

[16] P. Chamorro-Posada, G. S. McDonald, and G. H. C. New, J. Mod. Opt. 45, 111 (1998).

[17] P. Chamorro-Posada, G. S. McDonald, and G. H. C. New, J. Opt. Soc. Am. B 19, $1216(2002)$.

[18] P. Chamorro-Posada and G. S. McDonald, "Spatial Kerr soliton collisions at arbitrary angles," to be published in Phys. Rev. E.

[19] M. Lax, W. H. Louisell and W. B. McKnight, Phys. Rev. A 11, 1365 (1975).

[20] S. Chi and Q. Guo, Opt. Lett. 20, 1598 (1995).

[21] A. Ciattoni, P. Di Porto, B. Crosignani, and A. Yariv, J. Opt. Soc. Am. B 17, 809 (2000).

[22] V. Boucher, R. Barille and G. Rivoire, J. Opt. Soc. Am. B 20, 1666 (2003); L. Friedrich et al., Opt. Commun. 186, 335 (2000).

[23] M. Soljačić et al., Phys. Rev. Lett. 90, 254102 (2003); F. T. Hioe, Phys. Rev. Lett. 82, 1152 (1999).

[24] B. Crosignani, A. Yariv, and S. Mookherjea, Opt. Lett. 29, 1254 (2004); A. Ciattoni, B. Crosignani, S. Mookherjea, and A. Yariv, Opt. Lett. 30, 516 (2005); A. Ciattoni, B. Crosignani, P. Di Porto, J. Scheuer and A. Yariv, Opt. Express 14, 5517 (2006).

[25] P. Chamorro-Posada, G. S. McDonald, and G. H. C. New, J. Mod. Opt. 47, 1877 (2000).

[26] P. Chamorro-Posada and G. S. McDonald, Opt. Lett. 28, 825 (2003).

[27] R. Hirota, J. Math. Phys. 14, 805 (1973). 
[28] P. Chamorro-Posada, G. S. McDonald, and G. H. C. New, Opt. Commun. 192, 1 (2001).

[29] H. Goldstein, Classical Mechanics, $2^{\text {nd }}$ Ed. (Addison Wesley, Philippines, 1980), Chap. 12, p. 588.

[30] J. M. Christian, G. S. McDonald and P. Chamorro-Posada, "Helmholtz solitons in power-law media," submitted to J. Opt. Soc. Am. B.

[31] T. Carmon, M. Soljačcć and M. Segev, Phys. Rev. Lett. 89, 183902 (2002). 